\title{
A new edge detection approach via neutrosophy based on maximum norm entropy
}

\author{
Eser SERT ${ }^{\mathrm{a}, *}$, Derya $\mathrm{AVCI}^{\mathrm{b}}$ \\ a Department of Computer Engineering, Engineering and Architecture Faculty, Kahramanmaras Sutcu Imam University, K.Maras, Turkey \\ ${ }^{\mathrm{b}}$ Technical Sciences, Department of Computer Technology, Firat University, 23119 Elazig, Turkey
}

\section{A R T I C L E I N F O}

\section{Article history:}

Received 7 September 2017

Revised 11 July 2018

Accepted 11 August 2018

Available online 16 August 2018

\section{Keywords:}

Neutrosophy

Edge detection

Maximum entropy

FOM

PSNR

MSE

\begin{abstract}
A B S T R A C T
It is a quite important step to find object edges in applications such as object recognition, classification and segmentation. Therefore, the edge detection algorithm to be used directly influences the performance of these applications. In this study, a new edge detection method based on Neutrosophic Set (NS) structure via using maximum norm entropy (EDA-NMNE) is proposed. Many experts and intelligence systems, including the fuzzy system, do not satisfactorily succeed in resolving indeterminacies and deficiencies. However, in the NS approach, problems are solved by dividing them into True (T), False (F) and Indeterminacy (I) subsets. In addition, because the approach has a powerful algorithmic structure, NS's conditions with indeterminate and missing situations can be solved successfully. In this study, object edges can be found successfully with the proposed approach because edges of the object are considered as indeterminate. Thus, using the proposed EDA-NMNE, a strong intelligent expert system-based edge finding software was designed. In our study, 5 different object edge detection results were obtained by converting 5 different types of entropy into NS-based edge detection software. Thus, edge detection analysis has been performed by experimenting many types of entropy that have not been previously used for NS edge detection. The Metric Figure of Merit (FOM), Peak Signal-to-Noise Ratio (PSNR) analysis, mean square error (MSE) were used in this experimental study. Based on these results, highest FOM and PSNR values are obtained by using NORM entropy. In addition, the proposed edge detection approach were compared with Edge Detection Approach via Canny (EDA-C), Edge Detection Approach via Sobel (EDA-S), Edge Detection Approach via Variation-Adaptive Ant Colony Optimization (EDA-VAACO) and Edge Detection Approach via Active Contour without Edge (EDA-ACWE). FOM and PSNR tests have been used to evaluate the edge detection results obtained via 5 different methods. The findings demonstrated that the performance of the proposed edge detection approach is more successful compared to other methods.
\end{abstract}

(c) 2018 Elsevier Ltd. All rights reserved.

\section{Introduction}

Thanks to technological developments, the need to process visual data in digital media has increased, and edge detection has become one of the widely explored topics. Particularly in areas such as object recognition, medical image analysis, pattern recognition, image processing, edge information of object / objects is often required. Therefore, the correct orientation of the edges is an important factor in reaching efficient results.

Classical edge detection studies are performed based on Gradient and Laplacian operators. Canny (1986) and Laplacian of Gaussian (Sotak \& Boyer, 1989) basically use the Laplacian operator. Roberts (Rosenfeld, 1981), Prewitt (Seif et. al., 2010) and Sobel

\footnotetext{
* Corresponding author.

E-mail addresses: esersert80@gmail.com, esersert@ksu.edu.tr (E. SERT), derya2344@hotmail.com (D. AVCI).
}

(1990) use the Gradient operator In addition to Gradient and Laplacian based edge detection methods, wavelet (Tua \& Karstoft, 2015; Shih \& Tseng, 2005), neural network (Lu et al., 2003; Gua et. al., 2015), particle swarm optimization (Mahdi et. al., 2013), cuckoo search optimization (Gonzalez, et. al., 2016), ant colony optimization (Liu. \& Fang, 2015), Cellular Automata (Amrogowicz et al., 2016), Anisotropic Gaussian Kernels (Zhang et al., 2017b), and different edge detection approaches (Sun et al., 2016; Zhang et al., 2017a; Zareizadeh et. al., 2013) have also been proposed in the literature.

The Neutrosophic Set (NS) was introduced by Florentin Smarandache. It is based on the Neutrosophy theory and a new philosophical branch that has recently gained popularity (Smarandache, 2003). Superior performance is achieved by resolving vague and inconsistent situations using NS (AbdelBasseta et al. 2018). Since the problems in NS are analyzed by dividing them into True (T), Indeterminate (I) and False (F) subsets, 
three subset decision making processes are used. Because of all these features, NS solves problems more successfully compared to fuzzy logic (Smarandache, 2003; Alsmadi, 2016) as well as many expert and intelligence systems. In many real life situations such as biomedical, law, weather, stock exchange, and so on, the concept of neutrosophic logic can be used to solve indeterminacies (Kandasamy et al, 2005). Therefore, NS can be used as an expert decision support system in order to solve many problems. The use of NS yields successful results in applications such as Segmentation (Sert, 2018; Cheng et al., 2011; Guo \& Cheng, 2009; Zhang et al., 2010) and Edge Detection (Guo \& Şengür, 2014). In our study (Sert, 2018), 3D segmentation with norm-entropy-based NS was performed, and 3D modeling quality was increase.

Only a few studies can be found on NS-based edge detection in the literature. Dhar and Kundu (2017) proposed a text region segmentation method using NS and digital shearlet transform (DST). Anter and Hassenian (2018) proposed a segmentation method for abdominal CT liver tumor using NS, particle swarm optimization (PSO) and fast fuzzy C-means algorithm (FFCM). They benefited from NS-based pre-processing to improve the CT image and remove noise. Siri and Latte (2017) proposed a method which combined NS and Chan-Vese approaches. In this approach, image processing through $\mathrm{T}$, I and $\mathrm{F}$ subsets, which were obtained by converting CT scan image into NS domain, contributed to a successful liver segmentation. Guo et al. (2017) proposed a method that combines neutrosophic similarity score and active contour methods for automatic identification of myocardial echocardiography in left ventricle myocardial contrast.

In this study, a new edge detection approach based on NS structure via using maximum norm entropy (EDA-NMNE) is proposed. In the proposed approach, the picture is divided into $\mathrm{T}$, I and $\mathrm{F}$ subsets. Then, edge detection has been performed using these subsets. NS was used in this study since the edges in the images correspond to indeterminate situations and NS proved to be successful in the analysis of vague and inconsistent situations compared to many expert and intelligent systems (Dhar \& Kundu, 2017). Our main goal is to design a NS based expert and intelligent system which can be used for edge detection. We tested 5 different entropy types on 20 different images in order to reveal the impact of entropy used in NS on edge detection performance. Because the type of entropy used to identify the $\mathrm{T}$ and $\mathrm{F}$ subsets, which are the most important elements in edge detection with NS, is of vital importance, the type of entropy used directly affects the edge detection result. Therefore, in this study, it was necessary to conduct a detailed study on the notion of entropy. In addition, this method was compared with Edge Detection Approach via Canny (EDA-C) (Canny, 1986), Edge Detection Approach via Sobel (EDAS) (Sobel, 1990), Edge Detection Approach via Variation-Adaptive Ant Colony Optimization (EDA-VAACO) (Tian et al., 2011) and Edge Detection Approach via Active Contour without Edge (EDA-ACWE) (Chan \& Vese, 2001) methods in the literature to indicate the superiority of the EDA-NMNE method. As a result of all these process steps, industrial feasibility and methodological superiority of EDANMNE was proven.

The disadvantages of edge detection approaches using Gradient and Laplacian operator are noise sensitivity, illumination sensitivity and unadjusted parameters (Wu et. al., 2015; Thirumavalavan \& Jayaraman, 2016). Therefore, different edge detection approaches have so far been proposed to minimize the disadvantages of the above-mentioned edge detection approaches and increase their performance (Er-sen et al., 2009; Cho \& Cho, 1994; Xiao \& Hui, 2010). Fuzzy-based edge detection approaches have difficulties in creating fuzzy rules. The disadvantages of neural network based edge detection studies are long lasting training process and the dependence of their performance on the variety of training data. However, these disadvantages are minimized due to the above- mentioned features of NS-based edge detection. No other study has been carried out on the NS entropy analysis, which reveals the originality of the present study. Thus, this study aims to contribute to future studies on edge detection and segmentation via NS. The proposed EDA-NMNE proved to be superior to 4 different widely used edge detection approaches. The disadvantage of the proposed method is its intensive mathematical processes.

Ant colony optimization, which is an optimization algorithm based on swarm intelligence, was introduced by Dorigo et al. and inspired by the natural collective behavior of ants in the real world (Tian et al., 2011). EDA-VAACO, which is used for comparison purposes in this study, is an important technique obtained by making modifications in ant colony optimization. More information on EDA-VAACO can be found (Tian et al., 2011). EDA-ACWE, the fourth method used for comparison in the study, is one of the Regionbased methods and is based on the simplified Mumford-Shah function. This approach divides the image into an internal contour and an external contour globally by predicting the global brightness of the foreground and background (Chen et al., 2017). Further information on EDA-ACWE is available (Chan \& Vese, 2001).

The rest of the paper is organized as follows: 2. Theoretical Background is descried in Section 2. Experimental Results are presented in Section 3. Finally, Conclusions are given in Section 4.

\section{Theoretical background}

\subsection{Basics of neutrosophy}

The NS includes a tautological set (Smarandache, 2003), an intuitionistic set (Atanassov, 1986), a fuzzy set (Zadeh, 1965), a paraconsistent set (Priest, 2002) and a dialetheist set (Bruno, 2004), demonstrating that NS has solved the problems in a multidisciplinary way.

$E$ is an event, Non-E (N-E) is not $E$ and Anti-E (A-E) is the opposite of E. Neut-E (Ne-E) is named as neither $E$ nor A-E. For instance, if $\mathrm{E}=$ January, then A-E = December. N-E = February, March, April, May, June, July, August, September, October, November, December (any month except January). Ne-E = February, March, April, May, June, July, August, September, October, November (any month except January and December). In Neutrosophy, events are transformed into three subsets as T, I and F. T, I and F subsets are introduced as E, Ne-E and N-E respectively. I is an important subset in defining and resolving indeterminate situations in events. In NS, there are solutions to problems by performing operations on $\mathrm{T}$, I and F subsets. This subsets may be continuous or discrete parameters and may overlap as well. Moreover, they can be converted from one form into another (Smarandache, 2003). Because of these features, edge detection, segmentation and similar image processing applications with NS can be successfully performed (Alsmadi, 2016; Sert, 2018; Cheng et al., 2011; Guo \& Cheng, 2009; Zhang et al., 2010; Guo \& Șengür, 2014).

In this study, the image is divided into T, I and $\mathrm{F}$ subclasses in the Neutrosophic domain. There are objects / objects in the T subset, background in the F subset, and indeterminate situations such as noise, edge, color shifts in I subset (Sert, 2018). The Fig. 1 shows the flow diagram of the NS-based edge detection system. As can be seen in Fig. 1, the original image is initially converted to grayscale format and preprocessed, and the resulting preprocessed image is divided into T, F and I subsets in the neutrosophic domain. Then, Object (Obj), Edge (Edg) and Background (Bgd) are obtained. In the last step, the edges of the object / objects are detected.

\subsection{Preprocessing}

The input image is converted to the grayscale and then filtered by using anisotropic diffusion filter (ADF). ADF is a denoising tech- 


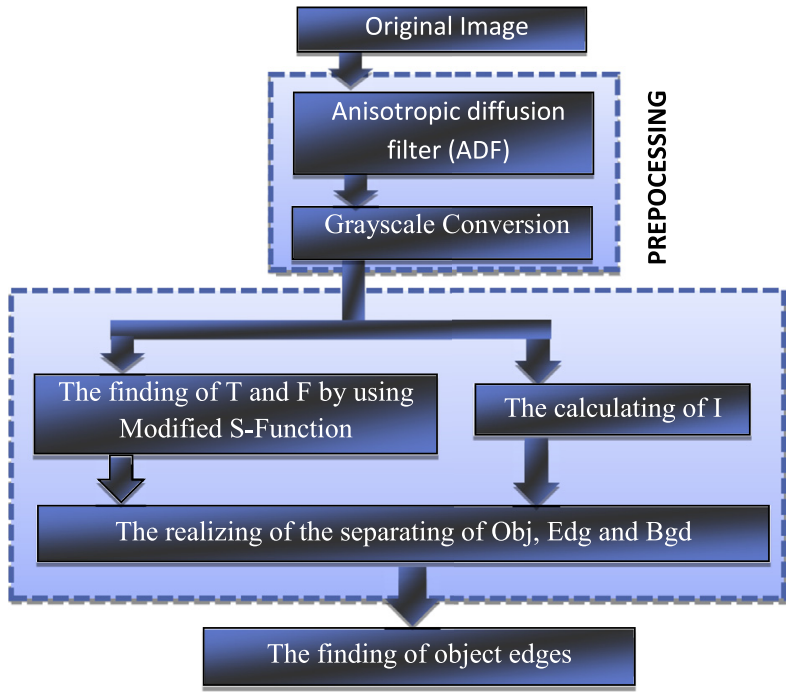

Fig. 1. The flow diagram of the proposed EDA-NMNE.

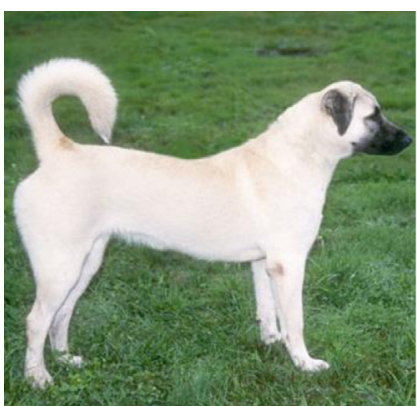

(a) Original image

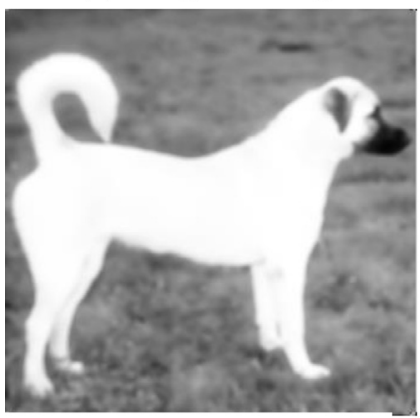

(c) T subset image

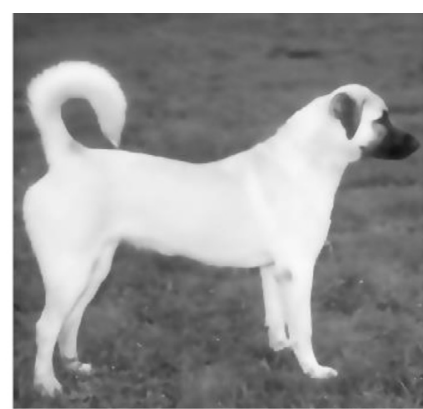

(b) Grayscale image

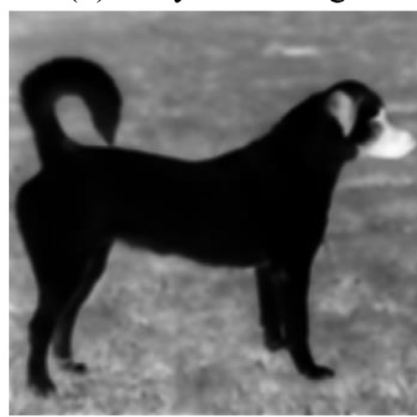

(d) F subset image
Fig. 2. Acquisition of images in $\mathrm{T}$ and F-domain with S-Function.

nique that provides superior performance especially in medical images (Michel-González et al., 2011). It also offers superior edge detection and segmentation on noisy images. Moreover, it preserves edge features of the images. Several types of filters have been experimented in the study, and ADF is preferred because it yields the best edge detection performance. Further information on this filter type is available in references (Weicker, 1997). In the second step of processing, this image is named Image Preprocessed $\left(I_{P}\right)$ after it is converted to grayscale filtered image.

\subsection{Process of obtaining $T$ and $F$ subsets}

In this work, the $\mathrm{T}$ and $\mathrm{F}$ subsets was found via performing the five different types of entropy in Zhang et al.'s (2010) method. The $\mathrm{S}$-function is an important tool used to represent the brightness level of the gray level (Cheng \& Chen, 1997). In here, S-function

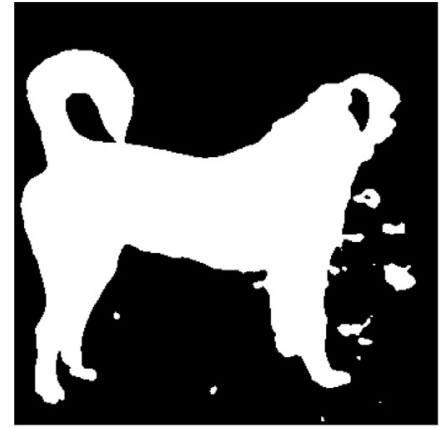

(a) Binarized - $\mathrm{T}$ image

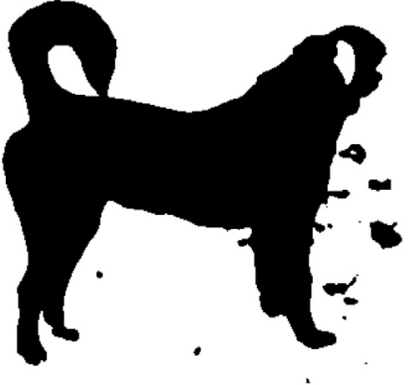

(b) Binarized - F image
Fig. 3. Acquisition of images of binarized $\mathrm{T}$ and $\mathrm{F}$ subsets.

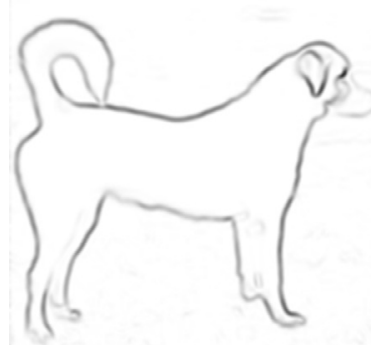

(a) Ho Image

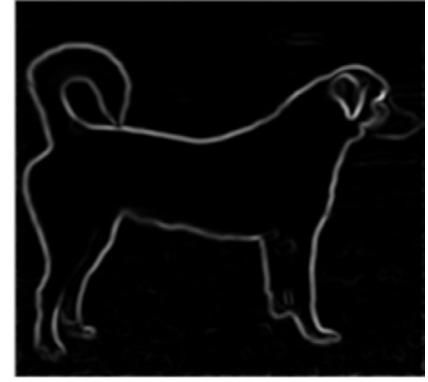

(b) I subset image
Fig. 4. The obtaining of I subset.

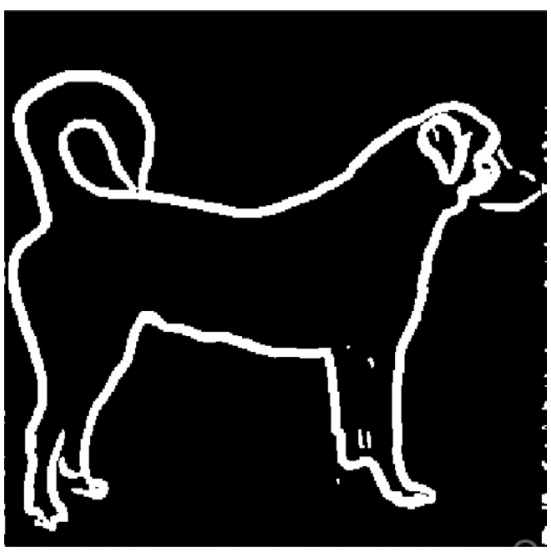

Fig. 5. $I_{\text {Binary }}$.

(Zhang et al., 2010; Cheng \& Chen, 1997; Cheng et al., 2004) is applied to $I_{P}$, as a result of, $\mathrm{T}$ subset is obtained. S-function are shown as follows:

$\mathrm{T}(\mathrm{i}, \mathrm{j})=\mathrm{S}\left(\mathrm{d}_{\mathrm{ij}} ; \mathrm{sp}_{1} ; \mathrm{sp}_{2} ; \mathrm{sp}_{3}\right)$

where $d_{i j}$ is the value of the intensity of the $(i, j)$ pixels of the image $\mathrm{I}_{\mathrm{P}} . \mathrm{sp}_{1}, \mathrm{sp}_{2}, \mathrm{sp}_{3}$ input parameters of $\mathrm{s}$-function determine the shape of the function. The operation of the s-function is given in Algorithm 1 (Zhang et al., 2010; Cheng \& Chen, 1997; Cheng et al., 2004):

\section{(1) Obtaining sp1 and sp3 Parameters:}

$\mathrm{sp}_{1}$ represents the peak value at left of the histogram. $\mathrm{sp}_{3}$ represents the peak value at the right of the histogram (Cheng et al., 2004). Detailed information on obtaining sp1 and $\mathrm{sp} 3$ parameters can be found in ref. (Zhang et al., 2010).

\section{(1) Obtaining sp2 Parameter:}




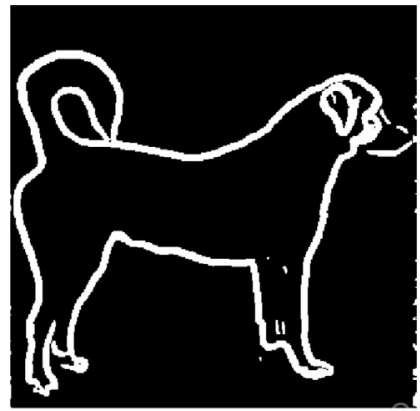

(a) I Binary $_{\text {B }}$

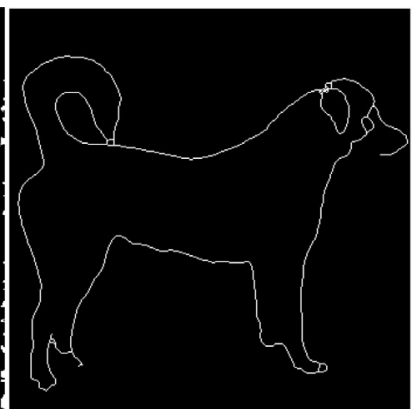

(b) $\mathrm{I}_{\text {Edge }}$

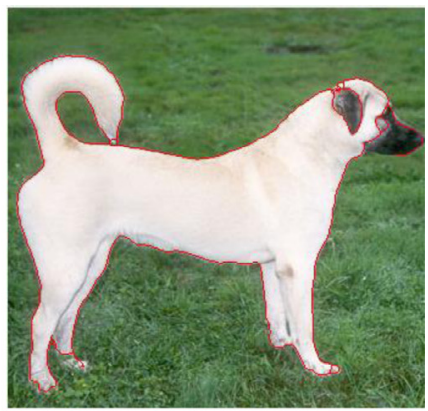

(c) IEdge Detected

Fig. 6. Images of edge detection process.

Algorithm 1

S-Function Algorithm.

$$
\begin{aligned}
& \text { 1. If }\left(\mathrm{d}_{\mathrm{ij}} \leq \mathrm{sp}_{1}\right) \\
& \text { 2. } \mathrm{T} \leftarrow 0 \\
& \text { 3. } \text { Else if }\left(\mathrm{sp}_{1} \leq \mathrm{dij} \leq \mathrm{sp}_{2}\right) \\
& \text { 4. } \mathrm{T} \leftarrow \frac{\left(d_{i j}-s p_{1}\right)^{2}}{\left(s p_{2}-s p_{1}\right)\left(s p_{3}-s p_{1}\right)} \\
& \text { 5. } \text { Else if }\left(\mathrm{sp}_{2} \leq \mathrm{dij}_{\mathrm{j}} \leq \mathrm{sp}_{3}\right) \\
& \text { 6. } \mathrm{T} \leftarrow 1-\frac{\left(d_{i j}-s p_{3}\right)^{2}}{\left(s p_{3}-p_{2}\right)\left(s p_{3}-s p_{1}\right)} \\
& \text { 7. } \text { Else if }\left(\mathrm{d}_{\mathrm{ij}} \geq \mathrm{sp}_{3}\right) \\
& \text { 8. } \mathrm{T} \leftarrow 0 \\
& \text { 9. } \text { End if }
\end{aligned}
$$

The maximum entropy principle given below is used for the calculation of the sp2 parameter (Cheng et al., 2004):

$$
H(\text { val })=\frac{1}{M x N} \sum_{j}^{N} \sum_{i}^{M} \text { EntropyType }(\mathrm{T})
$$

where $\mathrm{T}$ is output subset, which is obtained by the s-function. $\mathrm{M}$ and $\mathrm{N}$ represent the size of $\mathrm{T}$ image. Entropy Type corresponds to the selected entropy type. The system has a higher entropy value when the amount of information increases (Cheng \& Xu, 2000). The ideal optimal $\mathrm{sp}_{2}\left(\mathrm{sp}_{2 \mathrm{opt}}\right)$ value is obtained by trying all values from $\mathrm{sp}_{1}+1$ to $\mathrm{sp}_{3}-1$ (Cheng et al., 2004). Maximum $\mathrm{H}\left(\mathrm{d}_{\mathrm{ij}}\right)$ will be obtained based on $\mathrm{sp}_{2 o p t} . \mathrm{H}\left(\mathrm{d}_{\mathrm{ij}}\right)$ statement is given as follows (Zhang et al., 2010; Cheng \& Chen, 1997):

$$
\begin{aligned}
& \mathrm{H}_{\max }\left(\mathrm{d}_{\mathrm{ij}}, \mathrm{sp}_{1}, \mathrm{sp}_{2}, \mathrm{sp}_{3}\right) \\
& =\max \left\{\mathrm{H}\left(\mathrm{d}_{\mathrm{ij}} ; \mathrm{sp}_{1}, \mathrm{sp}_{2}, \mathrm{sp}_{3}\right) \mid \mathrm{d}_{\min }\right. \\
& \left.\leq \mathrm{sp}_{1} \leq \mathrm{sp}_{2} \mathrm{opt} \leq \mathrm{sp}_{3} \leq \mathrm{d}_{\max }\right\}
\end{aligned}
$$

\section{(1) Obtaining T and F Subset:}

In this study, different $\mathrm{H}(\mathrm{val})$ and $\mathrm{sp}_{2}$ opt values for each types of Shannon, norm, logarithmic energy, threshold and sure entropies are obtained. 5 different T-subsets are obtained by using five different $\mathrm{sp}_{20 p t}$ values in s-function. Because $\mathrm{T}$ subset is one of the most important factors in edge detection, the selected entropy type significantly influences the edge detection performance. As can be seen in the equation below, the F subset is obtained using the T subset:

$$
\mathrm{F}=1--\mathrm{T}
$$

In Fig. 2.a-d, original image, grayscale image, $\mathrm{T}$ subset image and $\mathrm{F}$ subset image are shown.

\section{(1) Obtaining Binary $\mathbf{T}$ and $\mathbf{F}$ Subset:}

Since $\mathrm{T}$ and $\mathrm{F}$ subsets obtained in the previous step are at the level of the grayscale, binary images are obtained by
Algorithm 2

The process of obtaining $\mathrm{T}$ and $\mathrm{F}$ subsets.
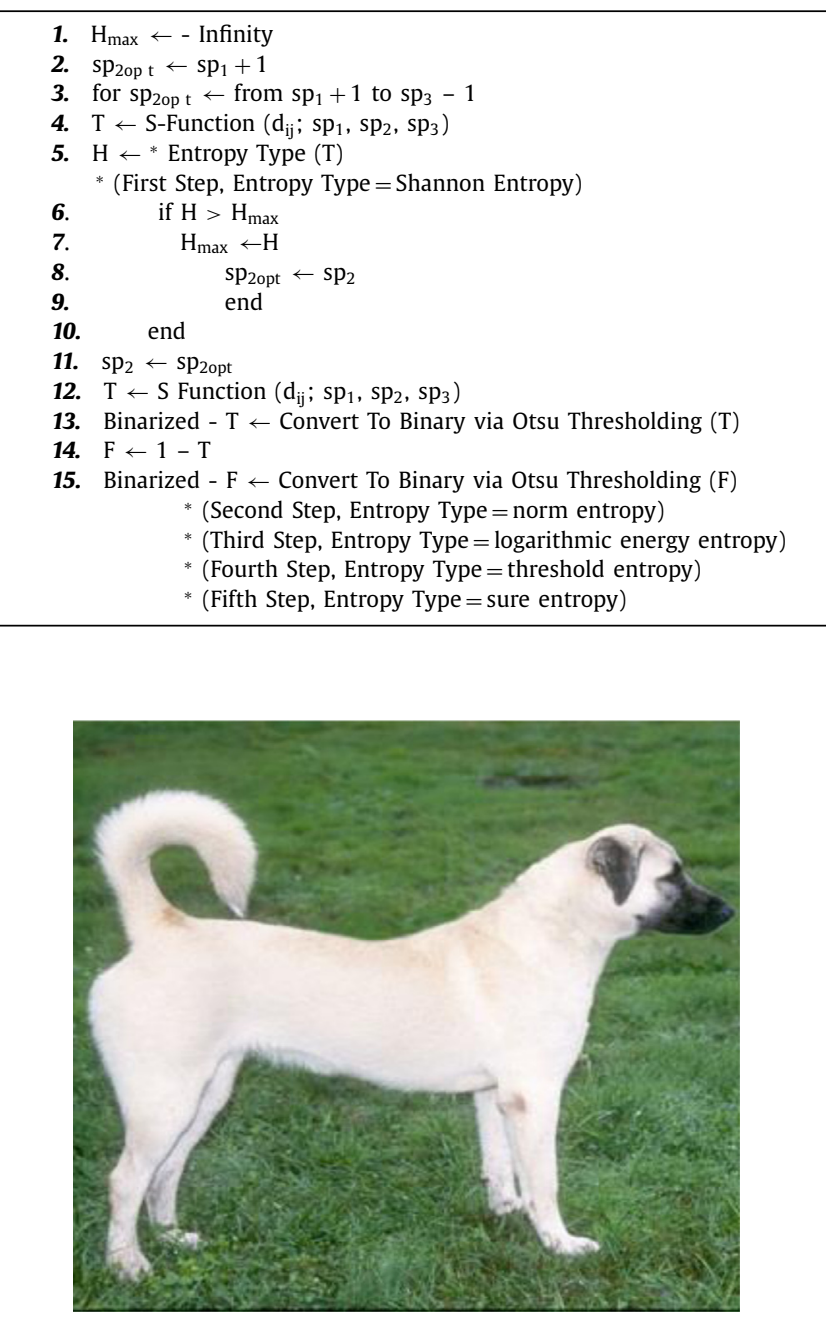

Fig. 7. The dog image used for edge detection analysis.

applying Otsu adaptive thresholding algorithm (Otsu, 1979) to these images. In image processing, Otsu's method automatically performs the image thresholding based on clustering (Sezgin \& Sankur, 2004). Detailed information on the method can be found in reference (Otsu, 1979). In Fig. 3.a-b, binarized $\mathrm{T}$ subset image and binarized $\mathrm{F}$ subset image are given. Information on the process of obtaining $\mathrm{T}$ and $\mathrm{F}$ subsets is summarized in Algorithm 2. 
Algorithm 3

The process of obtaining the I subset.

Step 1: Compute the standard deviation of the dij, which is the intensity at (i, j) coordinate of the $I_{P}$ image, and name it as stn_dv (i, j).

Step 2: Compute the discontinuity information of $I_{p}(i, j)$ using the sobel operator and name it as so_dsc (i, j).

Step 3: Calculate $\mathrm{H}$ parameter as follows:

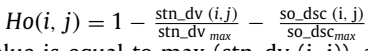

where (st_dev) $\max$ value is equal to $\max \left(\operatorname{stn} \_d v(i, j)\right)$, and (so_dsc) $\max$ value is max (so_dsc $(i, j)$ ).

Step 4: Calculate I subset as follows: I $(\mathrm{i}, \mathrm{j})=1-\mathrm{Ho}(\mathrm{i}, \mathrm{j})$

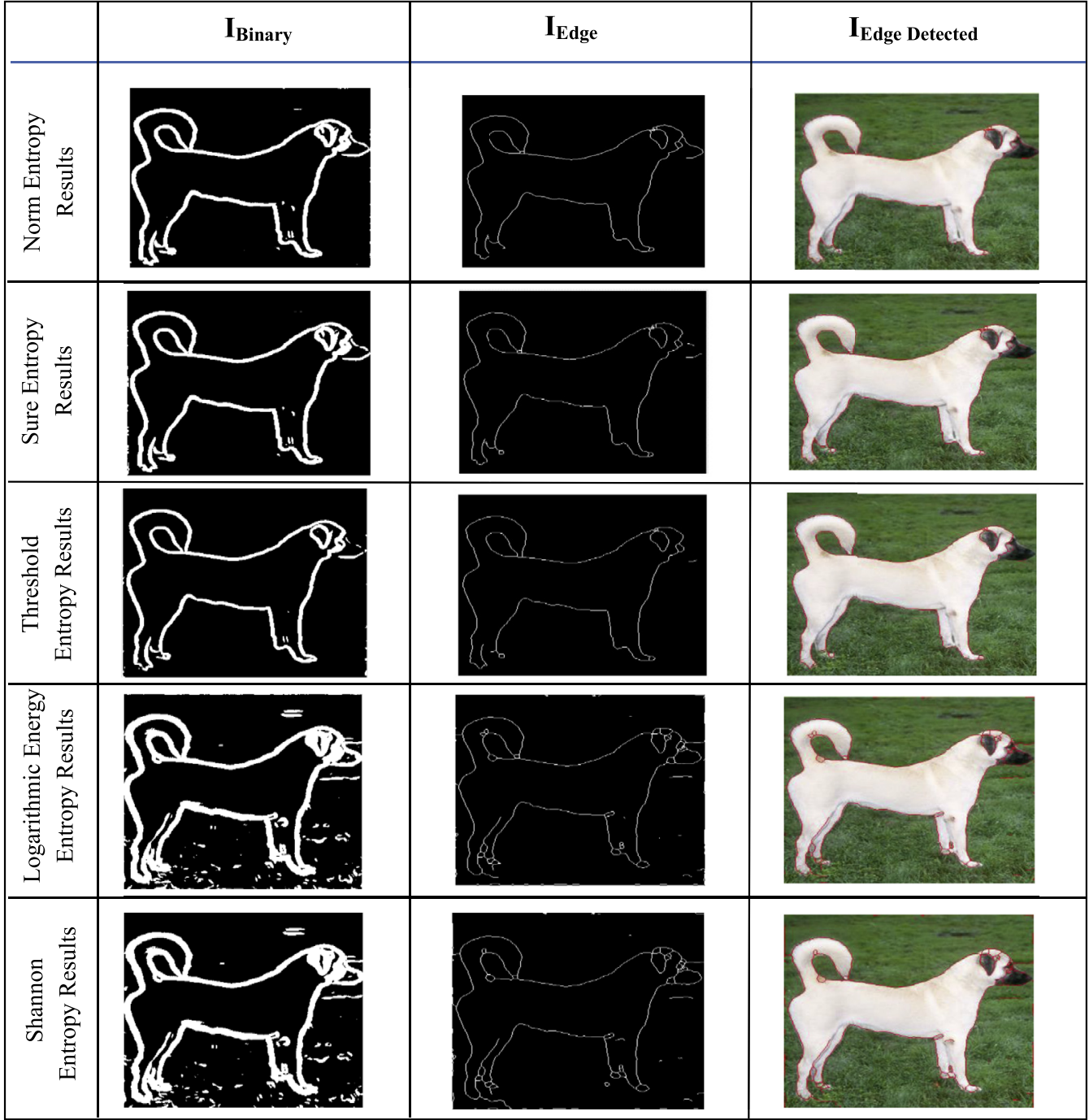

Fig. 8. The obtained edge detection results for dog image.

\subsection{Used entropy types}

In our study, the different types of the maximum entropy are used to find the $\mathrm{sp}_{2 \mathrm{opt}}$ Then, edge detection performances of these entropies are tested. Used entropy types are Shannon, norm, logarithmic energy, threshold and sure entropies, respectively. These entropy types and their equations are presented as follows:

Entropy (T) for Shannon Entropy $=-T_{(i, j)}^{2} \cdot \log _{2}\left(T_{(i, j)}^{2}\right)$

where $\log (0)=0$

Entropy (T) for Norm Entropy $=\left|T_{(i, j)}\right|^{p}$

where $1 \leq p<2$

Entropy(T)for Logarithmic Energy Entropy $=\log _{2}\left(T_{(i, j)}^{2}\right)$ where $\log (0)=0$

Entropy $(\mathrm{T})$ for Threshold Entropy $=E\left(T_{(i, j)}\right)$

where $\varepsilon$ is a positive threshold value. $\left|d_{(i, j)}\right|>$ $\varepsilon \Rightarrow E\left(T_{(i, j)}\right)=1$ and $\left|T_{(i, j)}\right| \leq \varepsilon \Rightarrow E\left(T_{(i, j)}\right)=0$

Entropy Type $(\mathrm{T})$ for Sure Entropy $=\min \left(T_{(i, j)}^{2}, \varepsilon^{2}\right)$

where $\left|T_{(i, j)}\right| \leq \varepsilon \Rightarrow \varepsilon$ isapositivethresholdvalue

\subsection{The obtaining of I subset}

Zhang et al.'s (2010) method was used to find I. One of the most important parameters is Homogenity (Ho), which is associated with local information and increases the value of this parameter in various situations such as edge regions, color transitions 


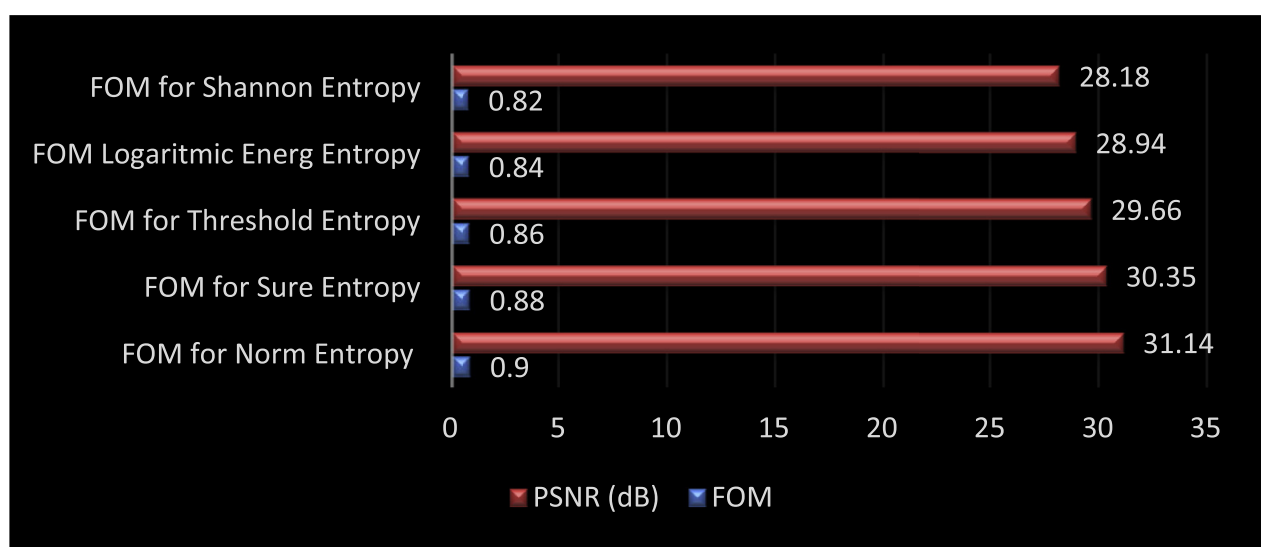

Fig. 9. The Results of FOM and PSNR for Dog Image.

Table 1

The edge detection results for different 20 images by using FOM and PSNR analysis.

\begin{tabular}{|c|c|c|c|c|c|c|c|c|c|c|}
\hline \multirow[t]{2}{*}{ Test No } & \multicolumn{2}{|c|}{ Norm entropy } & \multicolumn{2}{|c|}{ Sure entropy } & \multicolumn{2}{|c|}{ Threshold entropy } & \multicolumn{2}{|c|}{ Logarithmic energy entropy } & \multicolumn{2}{|c|}{ Shannon entropy } \\
\hline & FOM & PSNR (dB) & FOM & PSNR (dB) & FOM & PSNR (dB) & FOM & PSNR (dB) & FOM & PSNR (dB) \\
\hline 1 & 0,90 & 31,14 & 0,88 & 30,35 & 0,86 & 29,66 & 0,84 & 28,94 & 0,82 & 28,18 \\
\hline 2 & 0,92 & 32,78 & 0,89 & 30,95 & 0,84 & 29,11 & 0,78 & 23,97 & 0,77 & 24,62 \\
\hline 3 & 0,93 & 33,01 & 0,90 & 31,16 & 0,86 & 29,79 & 0,84 & 29,08 & 0,82 & 26,99 \\
\hline 4 & 0,95 & 33,99 & 0,92 & 32,23 & 0,91 & 31,81 & 0,88 & 30,53 & 0,86 & 29,78 \\
\hline 5 & 0,92 & 32,55 & 0,90 & 31,48 & 0,87 & 30,35 & 0,85 & 29,27 & 0,85 & 29,37 \\
\hline 6 & 0,96 & 34,20 & 0,95 & 33,40 & 0,92 & 32,30 & 0,90 & 31,48 & 0,89 & 31,08 \\
\hline 7 & 0,91 & 31,88 & 0,88 & 30,39 & 0,87 & 30,20 & 0,85 & 29,33 & 0,84 & 29,07 \\
\hline 8 & 0,88 & 30,15 & 0,87 & 29,98 & 0,85 & 29,48 & 0,84 & 29,42 & 0,83 & 27,85 \\
\hline 9 & 0,91 & 31,65 & 0,88 & 30,36 & 0,87 & 30,17 & 0,83 & 28,70 & 0,81 & 26,01 \\
\hline 10 & 0,92 & 31,73 & 0,90 & 31,45 & 0,88 & 30,74 & 0,88 & 30,52 & 0,85 & 29,33 \\
\hline 11 & 0,92 & 31,88 & 0,90 & 31,29 & 0,89 & 31,06 & 0,88 & 30,66 & 0,87 & 30,08 \\
\hline 12 & 0,95 & 33,88 & 0,93 & 32,50 & 0,92 & 32,45 & 0,92 & 32,29 & 0,90 & 31,41 \\
\hline 13 & 0,91 & 31,85 & 0,90 & 31,15 & 0,88 & 30,56 & 0,86 & 30,18 & 0,85 & 29,26 \\
\hline 14 & 0,96 & 33,58 & 0,94 & 32,35 & 0,92 & 32,01 & 0,91 & 31,94 & 0,90 & 31,47 \\
\hline 15 & 0,88 & 30,35 & 0,85 & 29,99 & 0,84 & 28,95 & 0,83 & 28,61 & 0,82 & 26,54 \\
\hline 16 & 0,96 & 33,98 & 0,95 & 33,40 & 0,94 & 33,12 & 0,91 & 31,87 & 0,89 & 30,89 \\
\hline 17 & 0,93 & 32,66 & 0,91 & 31,88 & 0,90 & 31,17 & 0,88 & 29,45 & 0,87 & 30,25 \\
\hline 18 & 0,89 & 30,94 & 0,85 & 29,24 & 0,85 & 29,51 & 0,83 & 28,70 & 0,81 & 25,35 \\
\hline 19 & 0,94 & 33,21 & 0,91 & 31,84 & 0,90 & 31,55 & 0,90 & 31,40 & 0,88 & 30,56 \\
\hline 20 & 0,93 & 32,92 & 0,91 & 31,76 & 0,88 & 30,58 & 0,87 & 30,13 & 0,86 & 30,11 \\
\hline
\end{tabular}

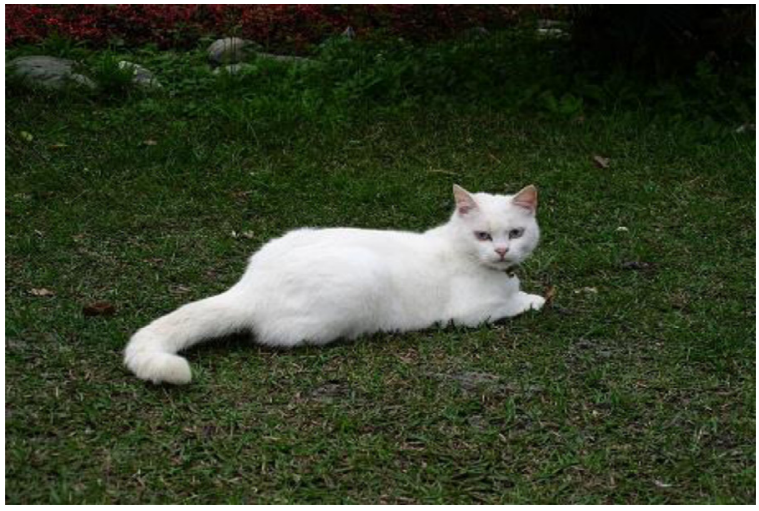

Fig. 10. The cat image used for edge detection analysis.

and background transitions in the image. The process of obtaining I subset is given in Algorithm 3 (Sert, 2018; Zhang et al., 2010). The standard deviation is important to achieve the contrast level of the pixels. To this aim, the standard deviation at the beginning of the algorithm is calculated. The discontinuity of the pixel $I_{P}(i, j)$ is explained by the edge value (Zhang et al., 2010). As can be seen in Algorithm 3, the discontinuity is computed by sobel operator. Obtained Ho and I subset images are shown in Fig. 4.

\subsection{Finding binary image}

In this step, Obj, Edg and Bgd variables are obtained. The methods of Zhang et al. (2010) were used to obtain these variables. This process is described in Algorithm 4.

Threshold $_{\text {IS }}$ parameter in Algorithm 4 is the threshold parameter for I. The value of this parameter is 0.04 at all analysis in this study. Threshold ${ }_{I S}$, Threshold TS $_{T S}$ and Threshold TF $_{\text {F }}$ parameters are used for Object, Edge and Background variables to be obtained during the segmentation process. The values of Threshold ${ }_{T S}$ and Threshold $_{S F}$ parameters are calculated by using Otsu Thresholding (Otsu, 1979) for T and F subsets, respectively. The Otsu's algorithm finds optimum threshold value, which separates object and background classes from each other. In this study, the Otsu's thresholding method for obtaining Threshold $_{T S}$ and Threshold SF $_{\text {F }}$ parameters was preferred compared to other thresholding methods because it yielded better results. Obtained $\mathrm{I}_{\text {Binary }}$ is shown in Fig. 5 .

\subsection{Edge detection process via EDA-NMNE}

Edge detection is performed using $\mathrm{I}_{\text {Binary }}$ and Original Image obtained in the previous step. Edge detection process is summarized in Algorithm 5. In the algorithm, morphological operations are performed on $\mathrm{I}_{\text {Binary }}$ and edges are transferred to $\mathrm{I}_{\mathrm{Edge}}$ variable. Then, $\mathrm{I}_{\text {Edge }}$ is superimposed on the Original Image to obtain the image ( $\left.\mathrm{I}_{\text {Edge Detected }}\right)$ whose edges are detected. 


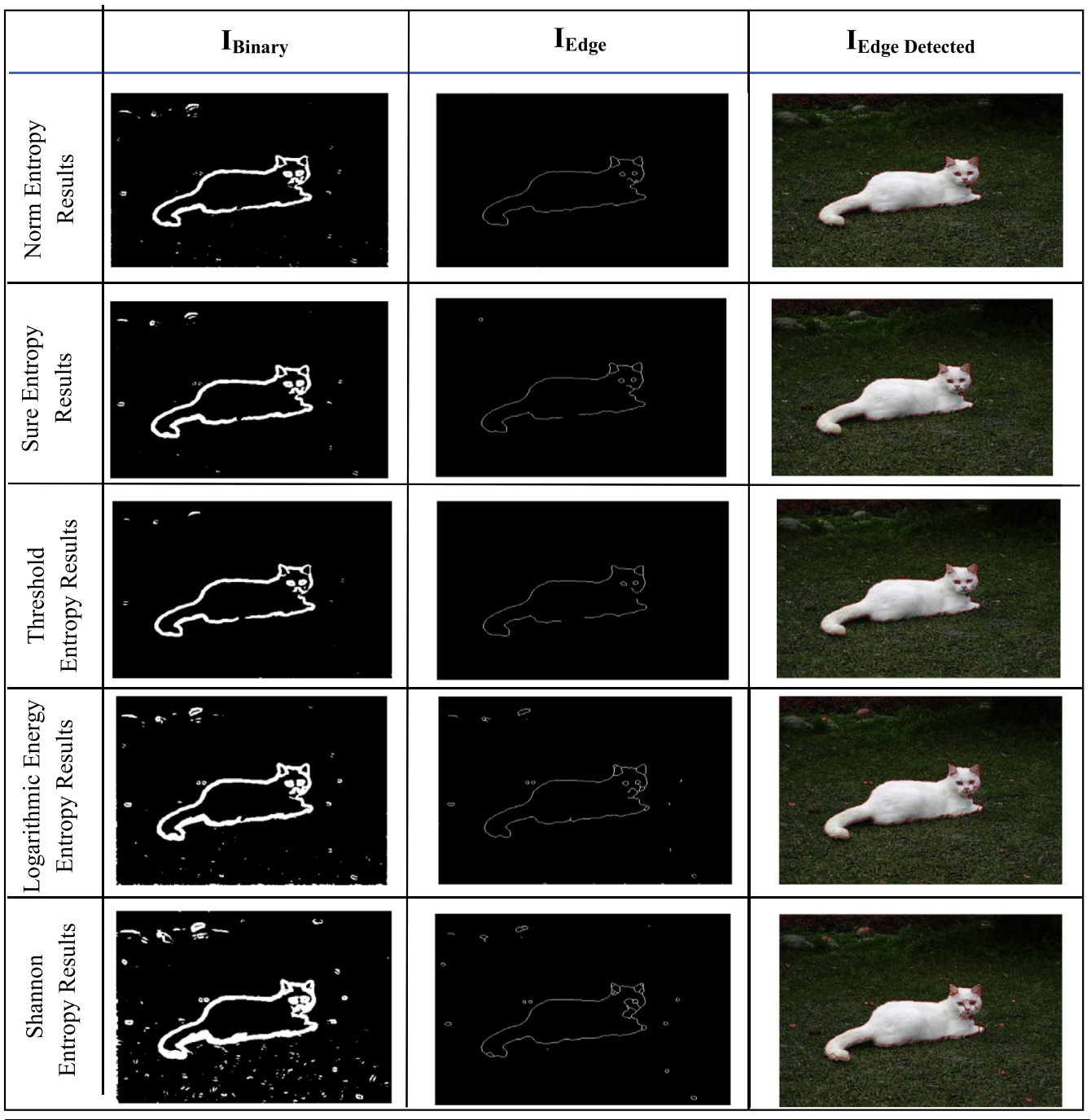

Fig. 11. The obtained edge detection results for dog image.

Table 2

Statistical results regarding the edge detection results.

\begin{tabular}{lllllllllll}
\hline & \multicolumn{2}{c}{ Norm entropy } & \multicolumn{2}{c}{ Sure entropy } & \multicolumn{2}{c}{ Threshold entropy } & \multicolumn{2}{c}{ Logaritmic energy entropy } & \multicolumn{2}{c}{ Shannon entropy } \\
& FOM & PSNR & FOM & PSNR & FOM & PSNR & FOM & PSNR & FOM & PSNR \\
\hline Maximum & 0,96 & 34,20 & 0,95 & 33,40 & 0,94 & 33,12 & 0,92 & 32,29 & 0,90 & 31,47 \\
Average & 0,92 & 32,42 & 0,90 & 31,36 & 0,88 & 30,73 & 0,86 & 29,82 & 0,85 & 28,91 \\
Minimum & 0,88 & \multirow{2}{*}{30,15} & 0,85 & 29,24 & 0,84 & 28,95 & 0,78 & 23,97 & 0,77 & 24,62 \\
\hline
\end{tabular}

As a result of the proposed edge detection approach, the images shown in Fig. 6 are obtained.

\section{Achievement measurement tools}

In this study, Metric figure of merit (FOM) proposed by Pratt (1978) and peak signal-to-noise ratio (Kaur \& Garg, 2011) (PSNR) are used to measure the success of edge detection approach. FOM is calculated as follows:

$\mathrm{FOM}=\frac{1}{\max \left(E_{D}, E_{A}\right)} \sum_{i=1}^{E D I_{A}} \frac{1}{1+d m^{2}(i)}$

where $E_{D}$ (Edge Detected $)$ are the number of pixels on the detected edge via edge detection approach, and $\mathrm{EI}_{\mathrm{A}}\left(\mathrm{Edge}_{\text {Actual }}\right)$ is the number of the actual edge pixels. $m(i)$ is the closest distance to $i$ th actual edge. $d$ is scaling parameter. FOM value is directly proportional to edge detection quality. FOM values of the most optimal edge detection methods are close to 1 . If this value decreases, the edge detection quality decreases. The used formula for PSNR analysis is given in Eq. (11). Moreover, mean square error (MSE) is used in order to determine edge detection quality in this experimental study. MSE formula is calculated as follows:

$\mathrm{MSE}=\sum_{i, j=1}^{S} \frac{\left[E_{A}(i, j)-E_{D}(i, j)\right]}{S^{2}}$

where $S$ is the size of the image.

$P S N R=10 \log _{10}\left(255^{2} / M S E\right)$

PSNR value is directly proportional to edge detection quality. In this experimental study, the highest PSNR value is nearly $35 \mathrm{~dB}$. If this value decreases, edge detection quality decreases. 


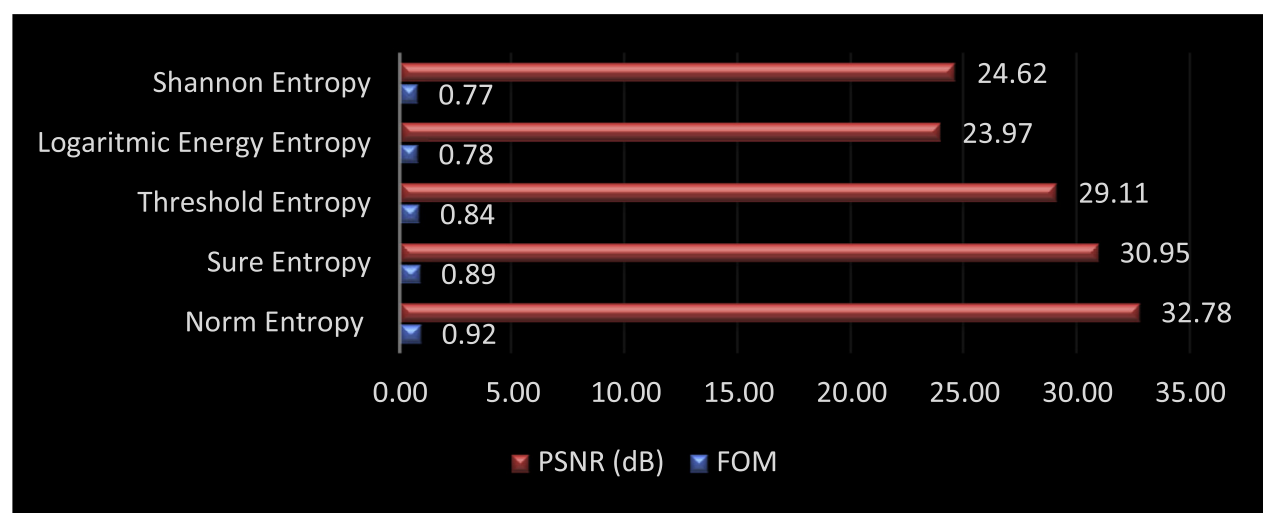

Fig. 12. The results of FOM and PSNR for cat image.

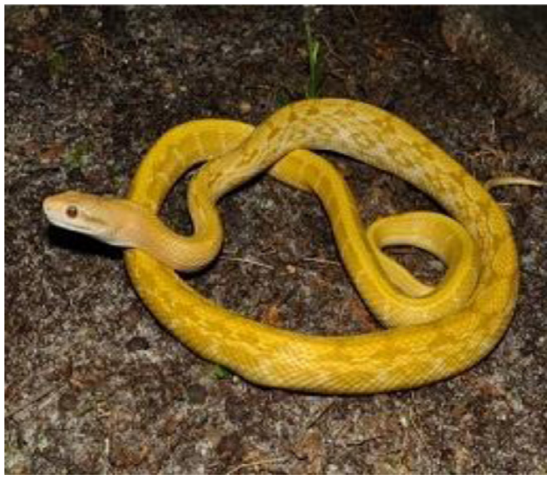

Fig. 13. The snake image used for edge detection analysis.

\section{Experimental results}

\subsection{Entropy performance test}

To test the edge detection performance of the norm entropy used in the proposed EDA-NMNE, EDA-NMNE is compared with NS based edge detection approaches with threshold, sure, logarithmic energy and Shannon entropy. For this process, 20 sample images which are difficult for edge detection are used. In the first test procedure, a dog image was used as shown in Fig. 7. The obtained edge detection results are given in Fig. 8. Moreover, FOM and PSNR analysis are performed based on $\mathrm{I}_{\text {Edge }}$ shown in Fig. 8 for the measurement of edge detection performance. Graphical representation of these FOM and PSNR analysis results are given in Fig. 9. The results of the analysis are presented under Test No. 1 in Table 1 . The highest edge detection performance was obtained by using Norm entropy, whereas the lowest edge detection performance was obtained in the Shannon entropy as shown in Fig. 9 and under Test No. 1 in Table 1.

The test procedures presented above were applied to a cat image shown in Fig. 10 and a snake image shown in Fig. 13. Accordingly, the results obtained from the analysis are presented in Fig. 11 for the cat image and Fig. 14 for the snake image. The FOM and PSNR analysis results of $\mathrm{I}_{\mathrm{Edge}}$ shown in Fig. 11 of the cat image are presented in Fig. 12, and the FOM and PSNR analysis results of $\mathrm{I}_{\text {Edge }}$ shown in Fig. 14 of the snake image are presented in Fig. 15. In addition, the analysis results obtained for the cat and snake images are presented in Test No. 2 and Test No. 3 in Table 1, respectively. As can be understood from both analyses, the EDA-NMNE displayed the highest FOM and PSNR results.

The same testing processes described in detail above were also applied to the images shown in Fig. 16.a, d, g, j. m. Because a lot of
Algorithm 4

Edge detection process.

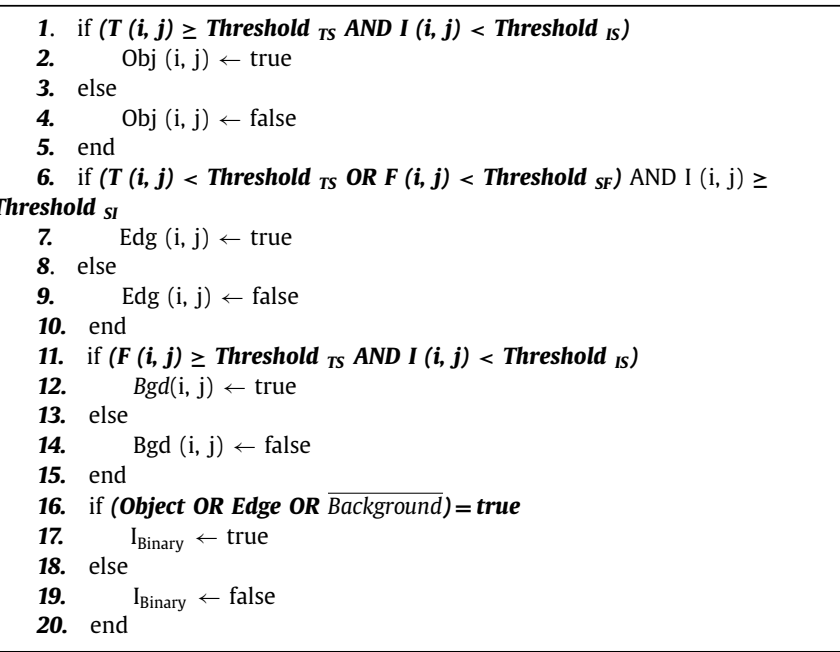

images were obtained at the end of these testing processes, EDANMNE method results are only presented in Fig. 16. FOM and PSNR analysis of the $\mathrm{I}_{\text {Edge }}$ in Fig. 16 are presented under Test No. 4, 5, 6, 7 in Table 1 . The results demonstrate that the highest success was displayed by Norm Entropy.

Following the analysis on eight different images, the same test was carried out on 12 different images. As a result, FOM and PSNR test results of $\mathrm{I}_{\mathrm{Edge}}$ were obtained as a result of 5 different entropies applied, and a total of 20 images are presented in Table 1. Fig. 17 and Fig. 18. illustrate graphical views of FOM and PSNR test results given in Table 1 . The results of statistical analysis on test results are presented in Table 2. As shown in Fig. 17, Fig. 18 and Table. 1, the highest edge detection performance was obtained by using Norm entropy, while the lowest edge detection performance was obtained in the Shannon entropy. From the statistical results in Table 2, the maximum, minimum and average values of FOM and PSNR results for the proposed approach are the highest. As a result of these tests, it was found out that the norm entropy used in EDA-NMNE is more successful compared to other entropies.

\subsection{The comparing applications of proposed EDA-NMNE and other edge detection methods}

EDA-NMNE was compared with EDA-C (Canny, 1986), EDA-S (Sobel, 1990), EDA-VAACO (Tian et al., 2011) and EDA-ACWE (Chan \& Vese, 2001) to measure the edge detection performance of the proposed EDA-NMNE. In the EDA-VAACO approach, edges are iden- 


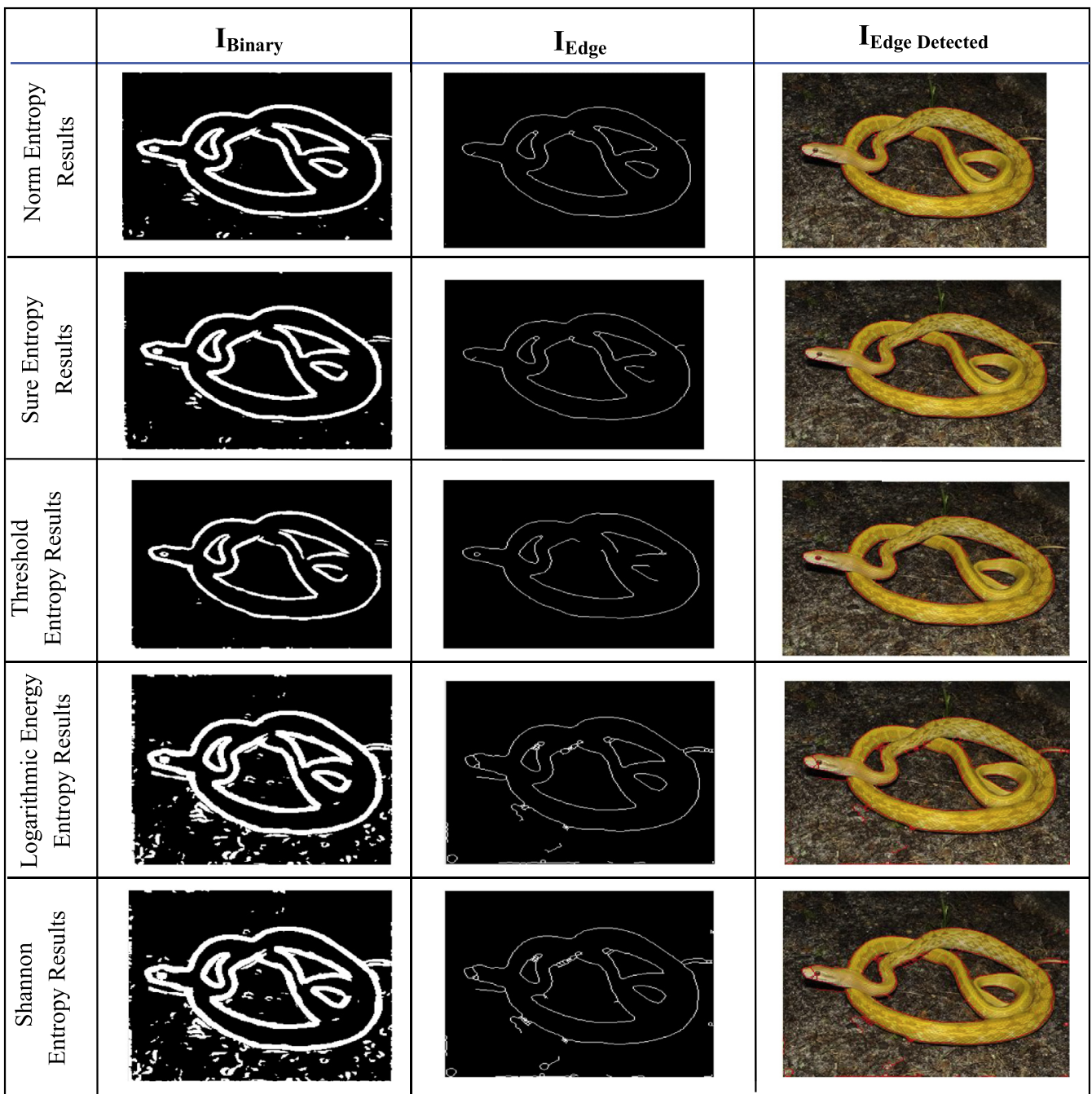

Fig. 14. The obtained edge detection results for horse image.

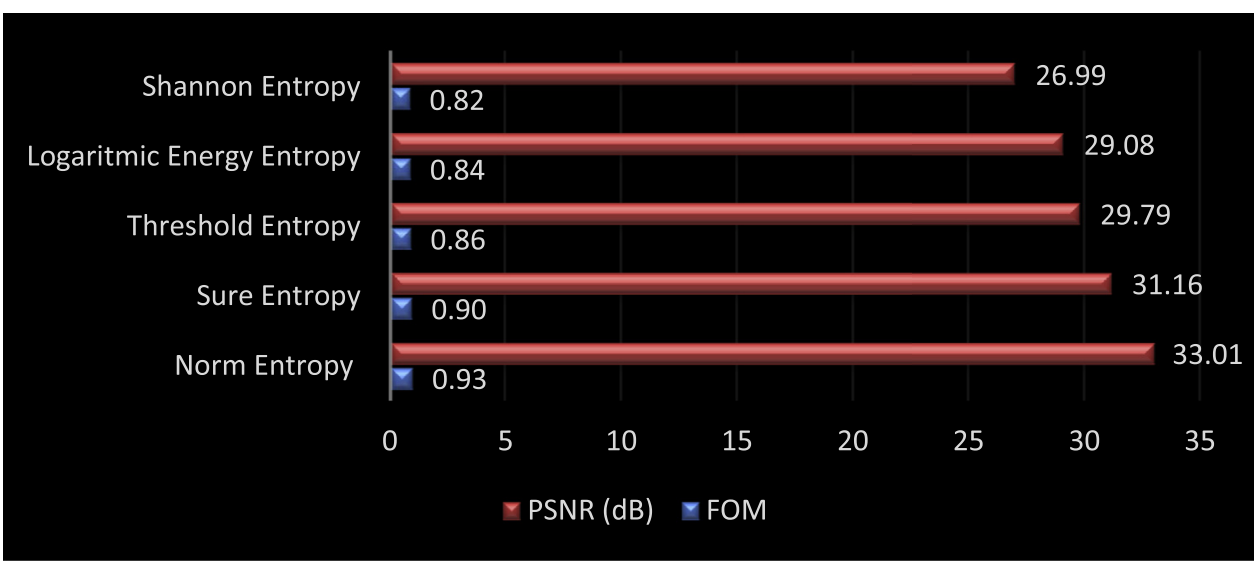

Fig. 15. The results of FOM and PSNR for snake image.

tified as thick lines. Therefore, morphological skeleton is applied to the output image. To measure the edge detection performance, 20 images which were used in the entropy test were employed, and these 5 edge detection approaches were applied to these images. Since the number of images is high, the IEDGE results of first 8 test images are presented in Fig. 19. As shown from the related figure, the most successful edge detection performance was obtained with the proposed EDA-NMNE. FOM and PSNR tests were performed to compare performances of $\mathrm{I}_{\mathrm{EDGE}}$ results for these 20 image. The graphical representation of FOM and PSNR results is shown in Fig. 20 and Fig. 21, respectively. The PSNR and FOM test results obtained with EDA-NMNE are higher compared to other four methods. In addition, statistical analyses were performed on FOM and PSNR results obtained via 5 edge detection approaches, and the results are presented in Table 3. As can be understood from Table 3, the maximum, arithmetic average and minimum val- 


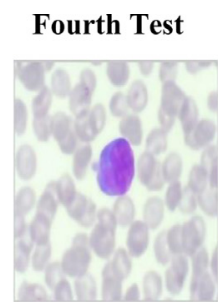

(a) Original imag

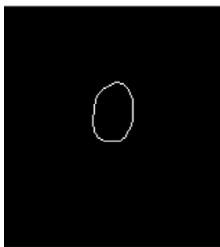

(b) $\mathrm{I}_{\mathrm{Edge}}$

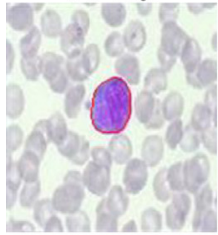

(c) IEdge Detected
Fifth Test

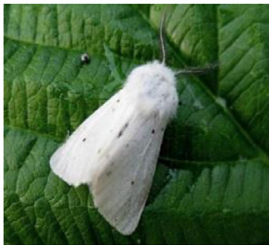

(d) Original image

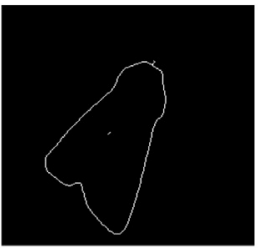

(e) $\mathrm{I}_{\text {Edge }}$

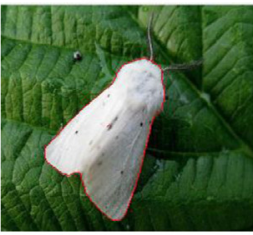

(f) $I_{\text {Edge Detected }}$

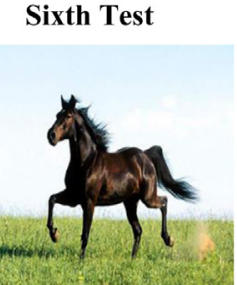

(g) Original image

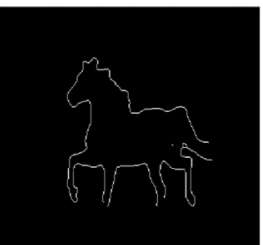

(h) $\mathrm{I}_{\text {Edge }}$

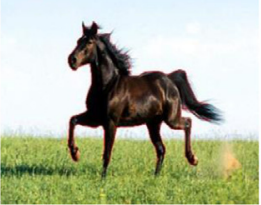

(i) $\mathrm{I}_{\text {Edge Detected }}$
Seventh Test

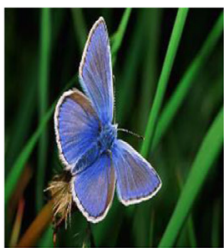

(j) Original image

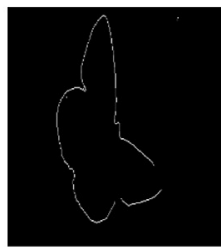

(k) $\mathrm{I}_{\mathrm{Edge}}$

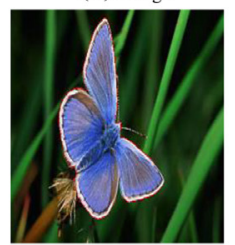

(1) $I_{\text {Edge Detected }}$
Eight Test

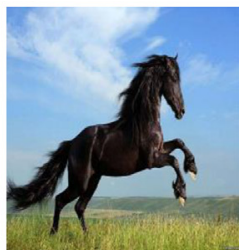

(m) Original image

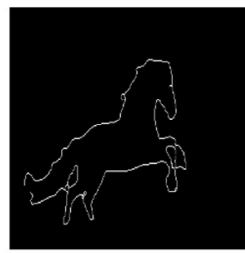

(n) $\mathrm{I}_{\text {Edge }}$

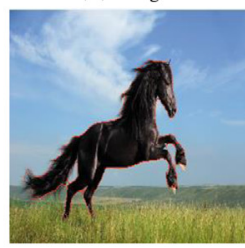

(o) $\mathrm{I}_{\text {Edge Detected }}$

Fig. 16. Test results.

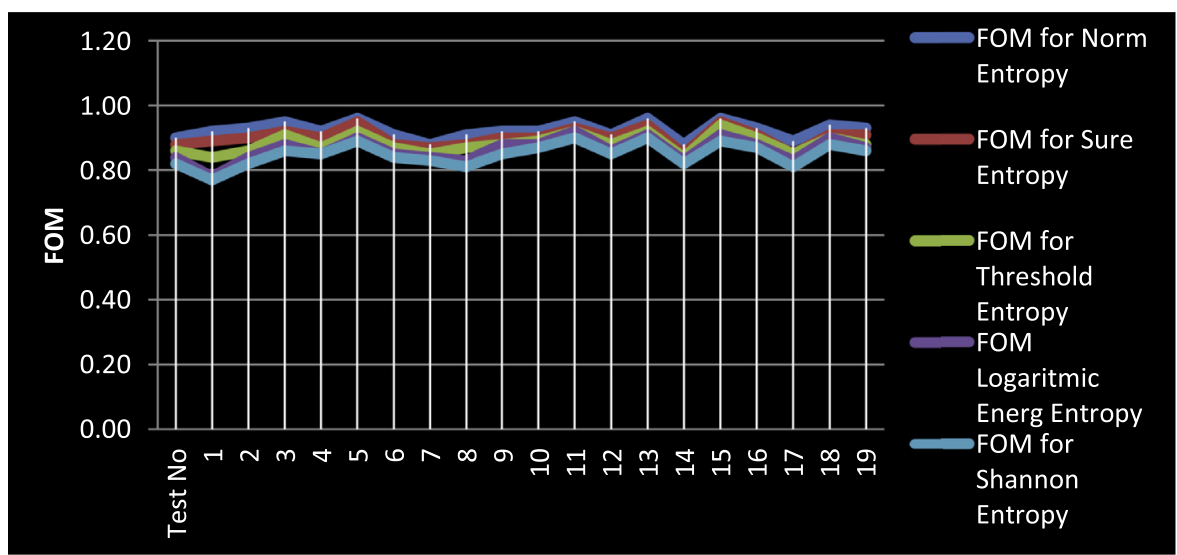

Fig. 17. The obtained FOM results regarding the edge detection results.

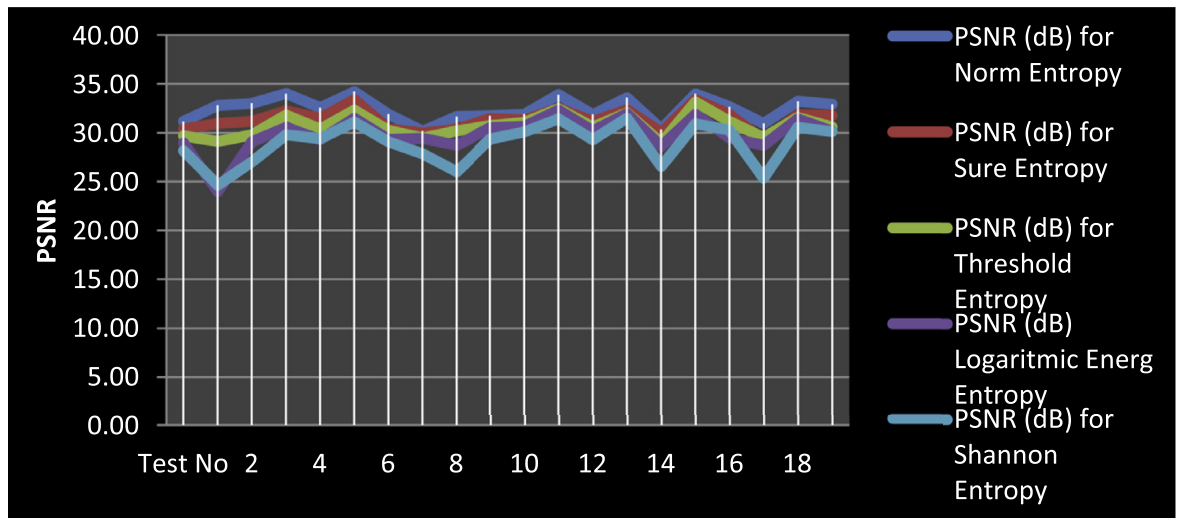

Fig. 18. The obtained PSNR results regarding the edge detection results. 


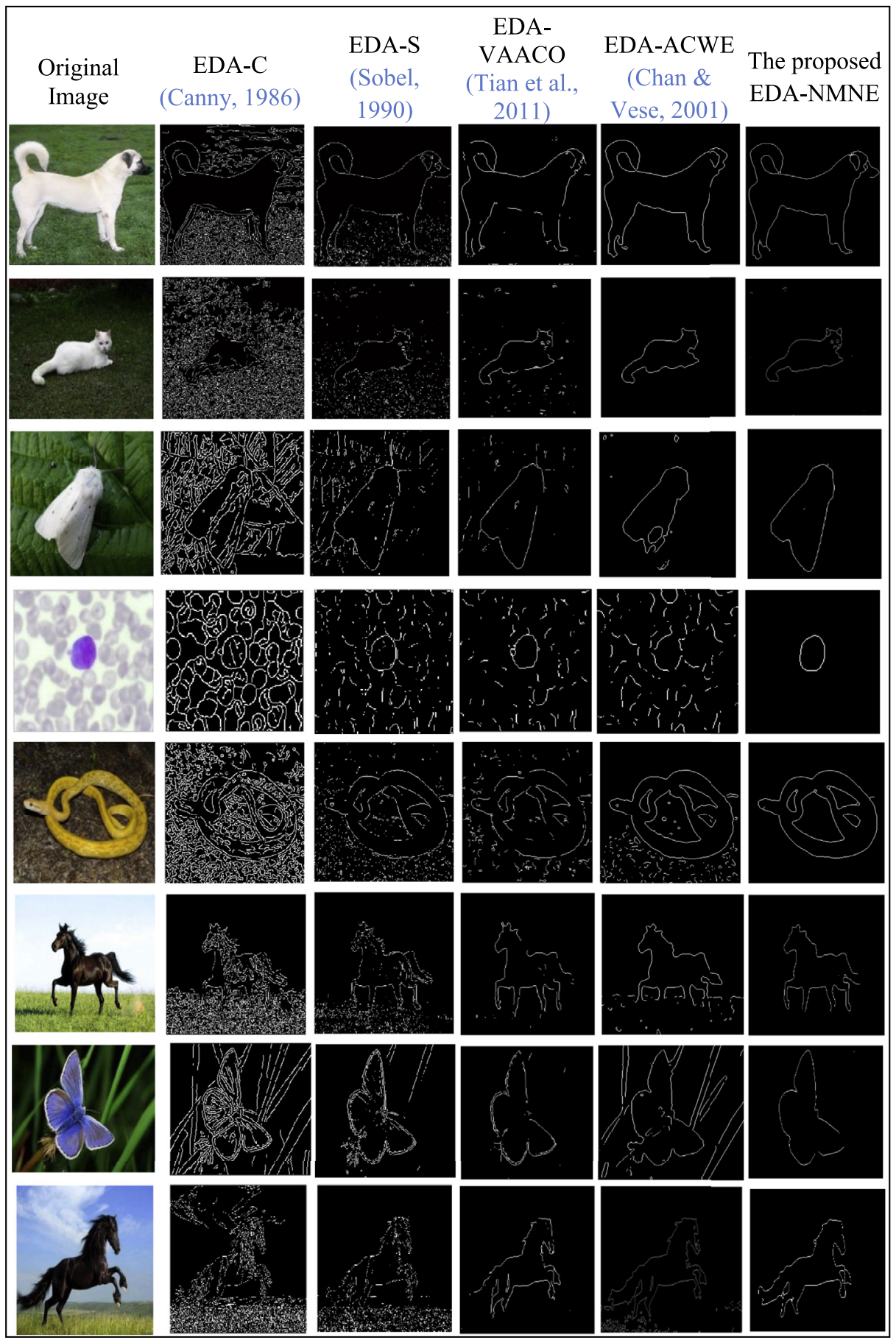

Fig. 19. Test results.

ues of the FOM and PSNR results obtained with EDA-NMNE are higher than other four methods. These results indicate that the proposed EDA-NMNE performs edge detection at a higher level compared to other methods.

As given in Table 2, average FOM and PSNR results for NORM entropy are 0.92 and 32.42 , respectively. Since these results are higher compared to those of other entropies, it can be suggested that the norm entropy in NS increases edge detection performance. As shown in Table 3, average FOM and PSNR results for EDA-NMNE are 0.92 and 32.42, respectively. These results are much higher compared to those of other edge detection algorithms. Because these results are much higher than the results of other edge detec- tion algorithms, one of the most important results of experimental study is that the proposed method detects object edges much more successfully compared to other methods.

\section{Conclusions and future work}

In this study, a new edge detection method based on NS structure is proposed using maximum norm entropy (EDA-NMNE). 100 different edge images $\left(\mathrm{I}_{\text {Edge }}\right)$ were obtained by trying 5 different types of entropy on 20 different images through NS-based edge detection approach. As shown in Table 2, which displays the statistical analysis performed on the FOM and PSNR test results, the most successful results were obtained by Norm entropy. Addition- 


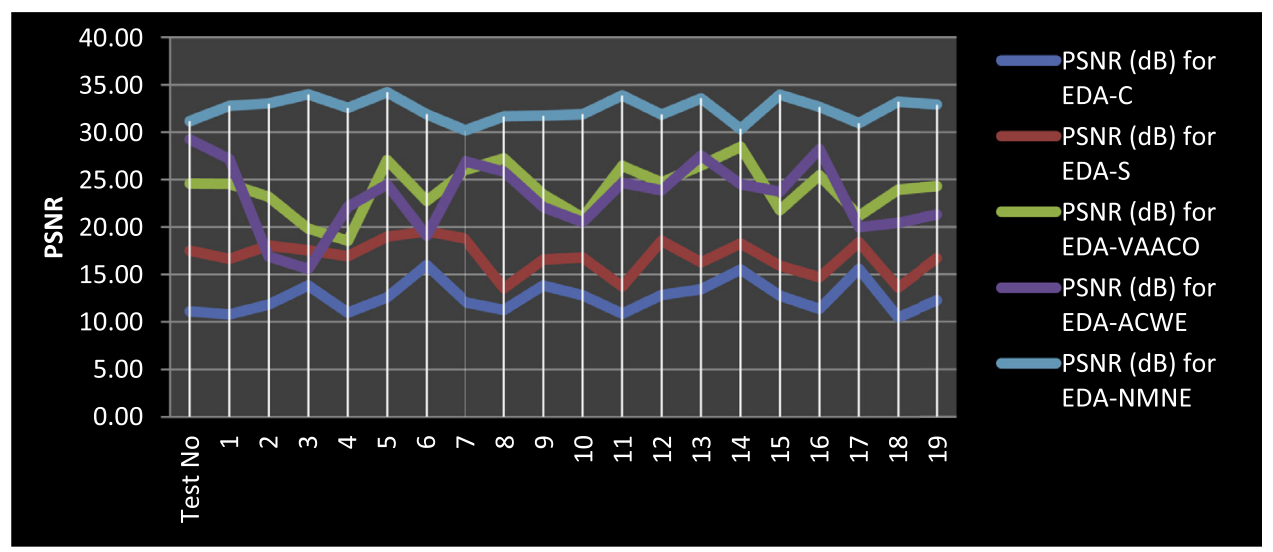

Fig. 20. The obtained PSNR results regarding the edge detection results.

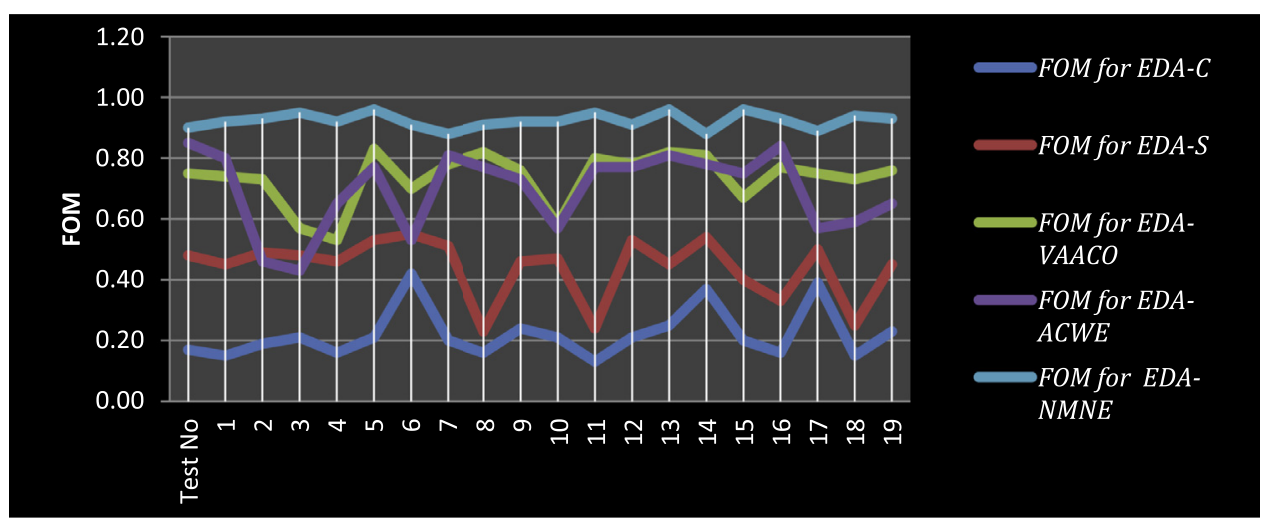

Fig. 21. FOM results for edge detection.

Table 3

Statistical results for edge detection.

\begin{tabular}{lllllllllll}
\hline & EDA-C & \multicolumn{3}{c}{ EDA-S } & \multicolumn{3}{c}{ EDA-VAACO } & \multicolumn{2}{c}{ EDA-ACWE } & \multicolumn{2}{c}{ EDA-NMNE } \\
& FOM & PSNR & FOM & PSNR & FOM & PSNR & FOM & PSNR & FOM & PSNR \\
\hline Maximum & 0,42 & 15,99 & 0,55 & 19,55 & 0,83 & 28,47 & 0,85 & 29,27 & 0,96 & 34,20 \\
Average & 0,22 & 12,61 & 0,44 & 16,85 & 0,73 & 24,05 & 0,70 & 23,21 & 0,92 & 32,42 \\
Minimum & 0,13 & 10,45 & 0,23 & 13,54 & 0,53 & 18,54 & 0,43 & 15,55 & 0,88 & 30,15 \\
\hline
\end{tabular}

Algorithm 5

Edge detection algorithm.

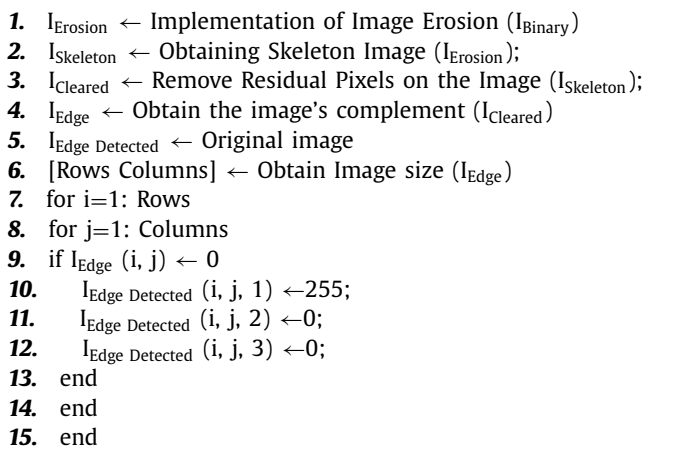

ally, proposed EDA-NMNE was compared with EDA-C, EDA-S, EDAVAACO and EDA-ACWE. According to the statistical analysis results of FOM and PSNR tests on the results of Table 3, FOM and PSNR results of EDA-NMNE are higher compared to other four methods. These results clearly demonstrates the superiority of the proposed method.
In the future, this study can be followed in four directions. Firstly, two directions are to explore new NS based edge detection and segmentation approaches using the theoretical and experimental experience gained in this study. Third direction is to perform 3D segmentation using NS. Fourth direction is to create NS based expert decision support systems that can be used in the healthcare sector, based on the success achieved by applying NS based edge detection approaches to biomedical data.

\section{Author contributions section}

Eser SERT: Conceptualization, Methodology, Software, Validation, Formal Analysis, Investigation, Writing - Original Draft, Writing - Review \& Editing, Visualization, Supervision, Project Administration, Funding Acquisition

Derya AVCI: Conceptualization, Methodology, Software, Resources, Data Curation, Writing - Review \& Editing

\section{References}

Abdel-Basseta, M., Manogaranb, G., Mohameda, M., \& Chilamkurt, N. (2018). Three-way decisions based on neutrosophic sets and AHP-QFD framework for supplier selection problem. Future Generation Computer Systems, 89, 19-30. Alsmadi, M. K. (2016). A hybrid fuzzy c-means and neutrosophic for jaw lesions segmentation. Ain Shams Engineering Journal (In Press). 
Amrogowicz, S., Zhao, Y., \& Zhao, Y. (2016). An edge detection method using outer Totalistic Cellular Automata. Neurocomputing, 214, 643-653.

Antera, A. M., \& Hassenian, A. E. (2018). Computational intelligence optimization approach based on particle swarm optimizer and neutrosophic set for abdominal CT liver tumor segmentation. Journal of Computational Science, 25, 376-387.

Atanassov, K. (1986). Intuitionistic fuzzy sets. Fuzzy Sets and Systems, 20(1), 87-96.

Bruno, W. (2004). Dialetheism, logical consequence and hierarchy. Analysis, 64(4), $318-326$.

Canny, J. A. (1986). A computational approach to edge detection. IEEE Transactions on Pattern Analysis and Machine Intelligence, 8(6), 679-698.

Chan, T. F., \& Vese, L. A. (2001). Active contours without edges. IEEE Transactions on Image Processing, 10, 266-277.

Chen, Y., Yue, X., Xu, R. Y. D., \& Fujita, H. (2017). Region scalable active contour model with global constraint. Knowledge-Based Systems, 120, 57-73.

Cheng, H. D., \& Chen, J. R. (1997). Automatically determine the membership function based on the maximum entropy principle. Information Sciences, 96(3-4), 163-182.

Cheng, H. D., \& Xu, H. (2000). A novel fuzzy logic approach to contrast enhancement. Pattern Recognition, 33(5), 809-919.

Cheng, H. D., Wang, J., \& Shi, X. (2004). Microcalcication detection using fuzzy logic and scale space approaches. Pattern Recognition, 37(2), 363-375.

Cheng, HD, Guo, Y., \& Zhang, Y. (2011). A novel image segmentation approach based on neutrosophic set and improved fuzzy c-means algorithm. New Mathematics and Natural Computation, 07(01), 155-171.

Cho, S. M., \& Cho, J. H. (1994). Thresholding for edge detection using fuzzy reasoning technique. In Proceedings of the IEEE international conference on computational science (pp. 1121-1124). IEEE.

Dhar, S., \& Kundu, M. K. (2017). Accurate segmentation of complex document image using digital shearlet transform with neutrosophic set as uncertainty handling tool. Applied Soft Computing, 61, 412-426.

Er-sen, L., Shu-long, Z., Bao-shan, Z., Yong, Z., Chao-gui, X., \& Li-hua, S. (2009). An adaptive edge detection method based on the canny operator. In Proceedings of the IEEE international conference environmental science and information application technology (pp. 265-269). IEEE.

Gonzalez, C. I., Pelin, M., Castro, J. R. C., O., \& Mendoza, O. (2016). Optimization of interval type-2 fuzzy systems for image edge detection. Applied Soft Computing, 47, 631-643.

Gua, J., Pana, Y., \& Wang, H. (2015). Research on the improvement of image edge detection algorithm based on artificial neural network. Optik, 126(21), 2974-2978.

Guo, Y., \& Cheng, HD. (2009). New neutrosophic approach to image segmentation. Pattern Recognition, 42(5), 587-595.

Guo, Y., Du, G.-Q., Xue, J.-Y., Xia, R., \& Wang, Y.-H. (2017). A novel myocardium segmentation approach based on neutrosophic active contour model. Computer Methods and Programs in Biomedicine, 142, 109-116.

Guo, Y., \& S Sengür, A. (2014). A novel image edge detection algorithm based on neutrosophic set. Computers and Electrical Engineering, 40, 3-25.

Kaur, B., \& Garg, A. (2011). Comparative study of different edge detection techniques. International Journal of Engineering Science and Technology (IJEST), 3(3), $1-5$.

Kandasamy, W.B.V., Smarandache, F., \& Kandasamy, K. (2005). Fuzzy and neutrosophic analysis of periyar's views on untouchability. USA: Hexis.

Liu, X., \& Fang, S. (2015). A convenient and robust edge detection method based on ant colony optimization. Optics Communications, 353, 147-157.

Lu, S., Wang, Z., \& Shen, J. (2003). Neuro-fuzzy synergism to the intelligent system for edge detection and enhancement. Pattern Recognition, 36(10), 2395-2409.

Mahdi, S., Mengjie, Z., \& Mark, J. (2013). A novel particle swarm optimisation approach to detecting continuous, thin and smooth edges in noisy images. Information Sciences, 246(10), 28-51.
Michel-González, E., Cho, M. H., \& Lee, S. Y. (2011). Geometric nonlinear diffusion filter and its application to X-ray imaging. BioMedical Engineering OnLine, 10(1), 47.

Otsu, N. (1979). A threshold selection method from gray-level histograms. IEEE Transactions on Systems, Man, and Cybernetics, 9(1), 62-66.

Pratt, WK. (1978). Digital image processing. USA: John Wiley \& Sons.

Priest, G. (2002). Paraconsistent logic. Dordrecht: Kluwer Academic Publishers.

Rosenfeld, A. (1981). The max Roberts operator is a Hueckel-Type edge detector. IEEE Transactions on Pattern Analysis and Machine Intelligence, 3(1), 101-103.

Seif, A., Salut, M. M., \& Marsono, M. N. (2010). A hardware architecture of Prewitt edge detection. In Proceedings of the IEEE conference on sustainable utilization and development in engineering and technology (STUDENT) (pp. 99-101). IEEE.

Sert, E. (2018). A new modified neutrosophic set segmentation approach. Computers \& Electrical Engineering, 65, 576-592.

Sezgin, M., \& Sankur, B. (2004). Survey over image thresholding techniques and quantitative performance evaluation. Journal of Electronic Imaging, 13(1), 146-165.

Shih, M.-Y., \& Tseng, D.-C. (2005). A wavelet-based multire solution edge detection and tracking. Image and Vision Computing, 23(4), 441-451.

Siri, S. K., \& Latte, M. V. (2017). Combined endeavor of Neutrosophic Set and Chan-Vese model to extract accurate liver image from CT scan. Computer Methods and Programs in Biomedicine, 151, 101-109.

Smarandache, F. (2003). A unifying field in logics neutrosophic logic. neutrosophy, neutrosophic set, neutrosophic probability. USA: American Research Press.

Sobel, J. (1990). Machine vision for three-dimensional scenes. New York: Academic Pres.

Sotak, G., \& Boyer, K. L. (1989). The Laplacian-of-Gaussian kernel: a formal analysis and design procedure for fast, accurate convolution and full-frame output. Computer Vision, Graphics, and Image Processing, 48(2), 147-159.

Sun, Q., Hou, Y., \& Tan, Q. (2016). A subpixel edge detection method based on an arctangent edge model. Optik, 127, 5702-5710.

Thirumavalavan, S., \& Jayaraman, S. (2016). An improved teaching-learning based robust edge detection algorithm for noisy images. Journal of Advanced Research, 7(6), 979-989.

Tian, J., Yu, W., Chen, L., \& Ma, L. (2011). Image edge detection using variation-adaptive ant colony optimization. In Transactions on computational collective intelligence (pp. 27-40). Springer. V.

Tua, G. J., \& Karstoft, H. (2015). Logarithmic dyadic wavelet transform with its applications in edge detection and reconstruction. Applied Soft Computing, 26, 193-201.

Weickert, J. (1997). A review of nonlinear diffusion filtering. Scale-space theory in computer vision. In Scale-space theory in computer vision, Lecture notes in computer science (pp. 1-28). Berlin: Springer. 1252.

Wu, Z., Lu, X., \& Deng, Y. (2015). Image edge detection based on local dimension: a complex networks approach. Physica A, 440, 9-18.

Xiao, W., \& Hui, X. (2010). An improved canny edge detection algorithm based on predisposal method for image corrupted by Gaussian noise. In Proceedings of the IEEE world automation congress (pp. 113-116). IEEE.

Zadeh, L. A. (1965). Fuzzy sets. Information and Control, 8(3), 353-383.

Zareizadeh, Z., Hasanzadeh, R. P. R., \& Baghersalimi, G. (2013). A recursive color image edge detection method using Green's function approach. Optik, 124, 4847-4854.

Zhang, M., Zhang, L., \& Cheng, H. D. (2010). A neutrosophic approach to image segmentation based on watershed method. Signal Processing, 90(5), 1510-1517.

Zhang, F., Qiao, N., \& Li, J. (2017a). A PCB photoelectric image edge information detection Method. Optik, 144, 642-646.

Zhang, W., Zhao, Y., Breckon, T. P., \& Chen, L. (2017b). Noise robust image edge detection based upon the automatic anisotropic gaussian kernels. Pattern Recognition, 63, 193-205. 